\title{
SmartOffice: Sala inteligente baseada numa arquitetura de sensores não intrusivos e técnicas de inteligencia computacional
}

\author{
Hyuri S. Maciel $^{1}$, André L. L. Aquino ${ }^{1}$ \\ ${ }^{1}$ Instituto de Computação - Universidade Federal de Alagoas (UFAL) \\ Caixa Postal 57072-9000 - Maceió - AL - Brasil \\ \{hyuril,alla\}@laccan.ufal.br
}

\begin{abstract}
Information about the occupation of an environment is necessary to implement advanced energy efficiency optimizations. In this sense, this work presents a monitoring and actuation system for intelligent buildings, detecting in a non-intrusive way whether the environment is occupied or not. The system consists of a low cost wireless network that monitors data of temperature, humidity, luminosity and the electric charge of the room while controlling both the illumination and temperature of the environment, a data analysis is performed to characterize the behavior of the environment. Finally, we obtain a $99.7 \%$ accuracy in detecting the environment using classification techniques in the sensed environmental data.
\end{abstract}

Resumo. Informações sobre a ocupação de um ambiente são necessárias para implementar otimizações avançadas de eficiência energética. Neste sentido este trabalho apresenta um sistema de monitoramento e atuação para prédios inteligentes, detectando de forma não intrusiva se o ambiente se encontra ocupado ou não. O sistema é composto por uma rede de sensores sem fio de baixo custo, que monitoram dados de temperatura, umidade, luminosidade e a carga elétrica da sala além de atuar no controle da iluminação e temperatura do ambiente, é realizada uma análise dos dados para caracterizar o comportamento do ambiente. Por fim obtemos uma taxa de acerto de 99,8\% na detecção do ambiente utilizando técnicas de classificação nos dados sensoreados.

\section{Introdução}

O contínuo crescimento geral da população fará com que 2,5 bilhões de novas pessoas passem a viver em áreas urbanas até 2050, que abarcarão mais de 6 milhões de habitantes dos 9,6 bilhões previstos [United Nations 2014]. Esse crescimento, tipicamente desordenado, causa um estresse na estrutura das cidades, devido ao aumento da demanda por recursos essenciais, como energia, água, serviços de saúde, segurança, lazer, entre outros [Banavar et al. 2011].

Alguns dos desafios tecnológicos são o desenvolvimento de inovações capazes de fornecer os recursos essenciais de forma mais eficiente a todos. Essas tecnologias devem estar diretamente relacionadas com o crescimento social, cultural e sustentável, de forma a melhorar a qualidade de vida e o convívio dos cidadãos. Para isso diversas Tecnologias da Informação e Comunicação (TICs) devem ser utilizadas para o desenvolvimentos das chamadas Cidades Inteligentes (CIs). 
O desenvolvimento de sistemas computacionais que percebam a intenção das pessoas que o utilizam seria o ideal, podendo assim, minimizar as tarefas e a necessidade de especificar tudo que o sistema faça por eles. Dessa maneira o contexto para o sistema aqui proposto é a habilidade de discriminar se o ambiente está ou não ocupado em um dado momento, assim permitir a atuação no sistema de iluminação e temperatura do ambiente, fazendo um uso mais eficiente da energia.

O aumento acentuado do consumo de energia elétrica nas cidades, embora possa refletir em um crescimento econômico e uma melhoria na qualidade de vida da população, também vem agregado de aspectos negativos. Um dos mais preocupantes é o esgotamento dos recursos utilizados para a geração de energia e seu impacto no meio ambiente. Necessitando de um grande investimento financeiro em novas fontes de geração elétrica e construção de novas usinas.

No Brasil os prédios residenciais e comerciais tiveram um aumento de $0.8 \%$ e $0.3 \%$ respectivamente no consumo de energia em $2018^{1}$, segundo a empresa de Pesquisa e Energia (EPE), no anuário de 2017 as classes residenciais e comerciais representam o segundo e terceiro maiores consumidores de energia no país, onde somados superam o primeiro lugar a indústria [EPE - Empresa de Pesquisa Energética 2017]. Uma maneira para conter a expansão do consumo elétrico sem comprometer a qualidade de vida da população e o desenvolvimento econômico e o estímulo do uso eficiente da energia.

O principal consumo de energia em edifícios está diretamente relacionados com sistemas de aquecimento e resfriamento, o crescimento no uso dos sistemas de HVAC (Healting, Ventilation and Air Condirioning - Aquecimento, Ventilação e ArCondicionado) é significativo. Neste sentido, por exemplo, o impacto de sistemas HVAC no uso da energia de edifícios nos países europeus representam cerca de $76 \%$ do consumo total [Nikolaou et al. 2012].

É possível determinar ocupação em salas utilizando modelos de classificação RF, CART, GBM e LDA com uma taxa de $98 \%$ de acurácia, quando utilizados apenas dois tipos de preditores (temperatura e luminância, luz e $\mathrm{CO}_{2}$ e relação luminância e umidade) [Candanedo and Feldheim 2016]. Mostrando-se uma abordagem promissora que por intermédio de técnicas computacionais podemos estimar a ocupação em salas de prédios inteligentes possibilitando gerar uma redução no consumo de energia elétrica.

Neste cenário, este trabalho trata de edifícios inteligentes com ênfase em eficiência energética, apresentando a criação de um SmartOffice, composto de uma rede de sensores e atuadores que realizam o monitoramento ambiental e atuação nas lâmpadas e ar-condicionado do ambiente, ademais o sistema identifica se o ambiente está ou não ocupado de forma não intrusiva, utilizando técnicas de aprendizado de máquina para esta finalidade.

O sistema de monitoramento utiliza equipamentos de baixo custo e poder computacional, o que não compromete sua eficiência. Para aplicação das técnicas de classificação utilizamos uma Raspberry ${ }^{2}$; para leitura dos dados e controle dos equipamentos utilizamos uma placa Arduino $\mathrm{UNO}^{3}$.

\footnotetext{
${ }^{1}$ http://epe.gov.br/pt

${ }^{2} \mathrm{https} / / / \mathrm{www}$. raspberrypi.org/

${ }^{3}$ https://www.arduino.cc/en/Guide/Introduction
} 
As principais contribuições deste trabalho são: (i) Criação de uma SmartOffice, um sistema de baixo custo para monitoramento ambiental e atuação nas lâmpadas e arcondicionado do ambiente; (ii) Análise dos dados sensoriados; (iii) Aplicação de técnicas de classificação para estimar se a sala ou está ou não ocupada. Com isso estima-se que o consumo de energia no ambientes vai reduzir, pois o sistema vai atuar de forma eficiente nos dispositivos.

\section{Embasamento teórico}

Para detecção de ocupação utilizamos os modelos RF, CART e K-NN, sendo esses uns dos métodos de classificação e regressão mais utilizados na literatura.

RF (Random forest - Floresta Aleatória), é um algoritmo de decisão e classificação de aprendizagem supervisionada, que cria uma floresta de um modo aleatório. RF tende a ser um dos algoritmos mais fáceis de usar e menos propenso a overfitting. Na maioria dos casos treinados com o método de bagging onde a ideia é gerar vários conjuntos de dados de treinamento por meio de amostragem dos dados originais do treinamento. Esses conjuntos de dados são utilizados para gerar outro conjunto de modelos usando um único algoritmo de aprendizado [Lantz 2015]. CART (Classification and Regression Tree - Árvore de Classificação e Regressão) é um dos algoritmos de árvore de decisão que foi proposto para realiza um processo binário, onde os nós pais são sempre divididos exatamente em dois nós filhos recursivamente, CART define um conjunto de regras que especifica a divisão de cada nó da árvore, associando cada nó terminal à uma classe que se deseja prever, realizando as previsões baseadas no valor médio de exemplos que atingem uma folha da árvore gerada [Lantz 2015].

Por fim, k-NN (k-nearest neighbors algorithm - k-ésimo vizinho mais próximo), embora seja uma das técnicas mais simples de classificação, ainda é amplamente utilizada. Sendo um classificador onde o aprendizado é baseado na relação de semelhança entre os vizinhos mais próximos. O conjunto de treinamento é formado por vetores n-dimensionais e cada elemento deste conjunto representa um ponto no espaço ndimensional [Lantz 2015].

Algumas das principais vantagens da utilização do K-NN: (i) Simples implementação. (ii) Não faz suposições sobre a distribuição de dados subjacente. (iii) seu treinamento é rápido, essas características se aplica muito bem ao nosso sistema onde todo processamento dos dados é realizados em computadores de baixo poder computacional, apresentados em 3. Algumas das desvantagens do K-NN: (i) requer a seleção de K um apropriado. (ii) dados ausentes requer um processamento adicional.

Descobrir os vizinhos mais próximos requer aplicar uma função de similaridade (distância) entre dois pontos. Seja $X=\left\{x_{1}, x_{2}, x_{3}, \ldots, x_{n}\right\}$ e $Y=\left\{y_{1}, y_{2}, y_{3}, \ldots, y_{n}\right\}$ dois pontos quaisquer no conjunto dos dados, temos as seguintes formas de realizar esse cálculo:

- Manhattan

$$
d(x, y)=\left\lfloor x_{1}-y_{1}\right\rfloor+\left\lfloor x_{2}-y_{2}\right\rfloor+\left\lfloor x_{3}-y_{3}\right\rfloor+\ldots+\left\lfloor x_{n}-y_{n}\right\rfloor
$$

- Minkowski

$$
d(x, y)=\left(\left\lfloor x_{1}-y_{1}\right\rfloor^{q}+\left\lfloor x_{2}-y_{2}\right\rfloor^{q}+\left\lfloor x_{3}-y_{3}\right\rfloor^{q}+\ldots+\left\lfloor x_{n}-y_{n}\right\rfloor^{q}\right)^{1 / q}
$$


- Distância Euclidiana, a mais utilizada

$$
d(x, y)=\sqrt{\left(x_{1}-y_{1}\right)^{2}+\left(x_{2}-y_{2}\right)^{2}+\left(x_{3}-y_{3}\right)^{2}+\ldots+\left(x_{n}-y_{n}\right)^{2}}
$$

A distância de Minkowski é a generalização das duas outras distâncias. Quando $q=1$, esta distância representa a distância de Manhattan e quando $q=2$, a distância Euclidiana. Se cada variável possuir um peso relativo à sua importância, a distância Euclidiana ponderada pode ser representada da seguinte forma:

$$
d(x, y)=\sqrt{w_{1}\left(x_{1}-y_{1}\right)^{2}+w_{2}\left(x_{2}-y_{2}\right)^{2}+w_{3}\left(x_{3}-y_{3}\right)^{2}+\ldots+w_{n}\left(x_{n}-y_{n}\right)^{2}}
$$

A letra $k$ é um termo variável implicando que qualquer número de vizinhos mais próximos poderia ser utilizado. Depois de escolher um $k$ adequado para o problema de classificação, o algoritmo necessita de um conjunto de dados de treinamento composto por uma amostra dos dados que foram classificados nas várias categorias, conforme rotulados através de uma variável nominal. Então, para cada registro não rotulado no conjunto dos dados de teste, $\mathrm{k}-\mathrm{NN}$ identifica os $k$ registros mais próximos, em similaridade, entre os dados que foram treinados. A distância dos dados de teste não rotulada é atribuída à classe da maioria dos $k$ vizinhos que estão mais próximos.

A decisão de quantos vizinhos usar para k-NN vai determina quão bem o modelo será generalizado para dados futuros. Em contrapartida, o ajuste inadequado dos dados de treinamento pode gerar um problema de overfitting. A escolha de um $k$ grande reduz o impacto ou a variação causada por dados ruidosos, porém, pode ignorar o reconhecimento de padrões pequenos que sejam importantes para o problema da classificação.

Entre os trabalhos relacionados, e os citados neste trabalho sobre detecção de ocupação em ambientes restritos como salas de prédios, temos o estudo de [Ghai et al. 2012] que apresenta uma abordagem para detectar ocupação utilizando fontes de contexto como crachás de identificação e WI-FI, detectando com uma precisão de $90 \%$ a ocupação. Outro trabalho interessante é [Kleiminger et al. 2013] que coletaram dados sobre o consumo elétrico de cinco residências, aplicando técnicas de classificações comuns, alcançaram precisão de mais de $80 \%$ na detecção de ocupação dos ambientes monitorados.

\section{Sistema de monitoramento e controle}

O sistema proposto, SmartOffice, considera o monitoramento de dados ambientais, o controle do sistema de iluminação, refrigeração e a detecção da ocupação de pessoas no ambientes. O sistema pode ser facilmente implementado em prédios públicos, laboratórios, salas de reuniões, de forma que as predições da ocupação é realizada pelos dados sensoriados quando aplicadas as técnicas de classificação.

O protótipo é composto por uma rede de sensores sem fio (RSSF), que integra sensores de monitoramento ambiental, atuadores e microcontroladores. O objetivo é realizar coleta dos dados e a troca de informação entre os sensores distribuídos no ambiente. A RSSF contém inicialmente três nós, que são:

1. Nó 1 denominado (SINK), é responsável por receber os dados dos sensores; aplicar as técnicas de classificação para detectar a ocupação no ambiente; e realizar a disseminação de informação para os outros nós da RSSF. 
2. Nó 2, é responsável pelo monitoramento da temperatura, umidade e luminosidade, também realiza a atuação no sistema de iluminação e refrigeração.

3. Nó 3, coleta informação do consumo elétrico do ambiente onde o sistema está instalado.

\subsection{Comunicação}

A comunicação entre os dispositivos é realizada por intermédio de rádio frequência (RF), evitando acesso direto com a internet. Entre os diversos tipos de soluções comerciais para comunicação de sistemas embarcados, avaliamos a comunicação por RF com o módulo NRF24L01 [Nordic semiconductor 2008], sendo a melhor escolha para nossa aplicação, devido ao baixo custo e praticidade para realizar a comunicação, possibilitando o suporte a vários tipos de embarcados.

O módulo NRF24L01 Wireless transceiver [Nordic semiconductor 2008] fabricado pela Nordic, possibilita uma comunicação na faixa de frequência de $2.4 \mathrm{GHz}$ entre dispositivos como Arduino, PICs, Raspberry, Beaglebone, entre outros, facilmente programável através de uma interface SPI. Seu alcance pode chegar a 10 metros em ambientes internos e 50 metros em campo aberto. Possui uma tensão de alimentação entre 1.9 a 3.6 volts e, corrente de alimentação de $1 \mathrm{~mA}$, com velocidade de operação na faixa de $2 \mathrm{Mbps}$, modulação e comunicação de multiponto com até 125 canais, o que se torna ideal para nossa aplicação.

\subsection{Dispositivos da RSSF}

A figura 1 representa o nó central SINK da RSSF, composto por uma Raspberry PI 3 Model B+, com processador Broadcom BCM2837B0 64 bits ARM Cortex-A53 QuadCore, clock 1.4GHz, memória RAM: 1GB, adaptador WiFi $802.11 \mathrm{~b} / \mathrm{g} / \mathrm{AC} 2.4 \mathrm{GHz}$ e $5 \mathrm{GHz}$ integrado, integrado também um módulo de rádio NRF29L01.

O nó SINK contém um sistema operacional MATE que fornece um ambiente Gnu/Linux, propiciando a instalação dos pacotes e bibliotecas necessárias utilizadas para nossa aplicação.

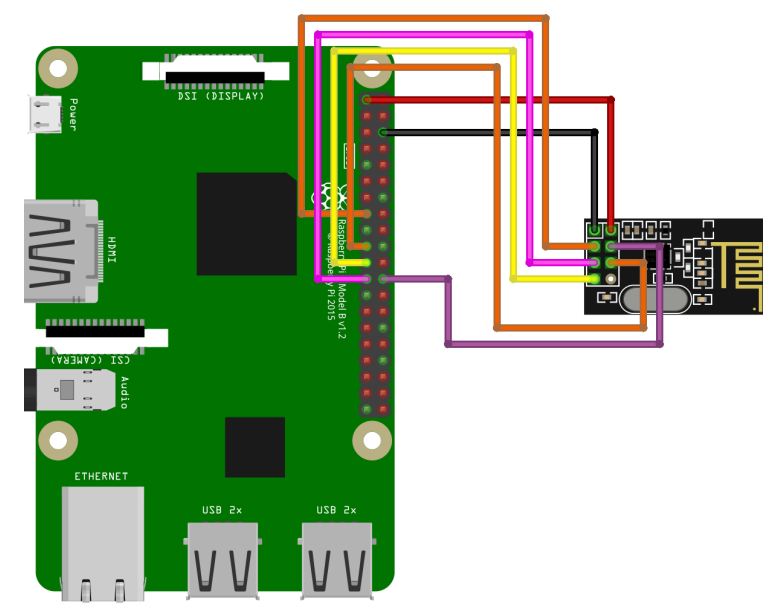

Figura 1. Protótipo nó SINK, utilizando uma RaspBerry Pi 3 e um módulo NRF24L01 para comunicação. 
O nó 1 representado pela figura 2 é composto por um Arduino UNO, um sensor DHT11 [Ltd. 2010] para o monitoramento da temperatura e umidade, um sensor de luminosidade LDR [Technologies 2010] que monitora a luminância do ambiente, quatro relés que atuam como chaves magnéticas para ligar e desligar as lâmpadas, um transmissor infravermelho para controlar o ar-condicionado da sala, e um módulo de rádio NRF24L01 para comunicação.

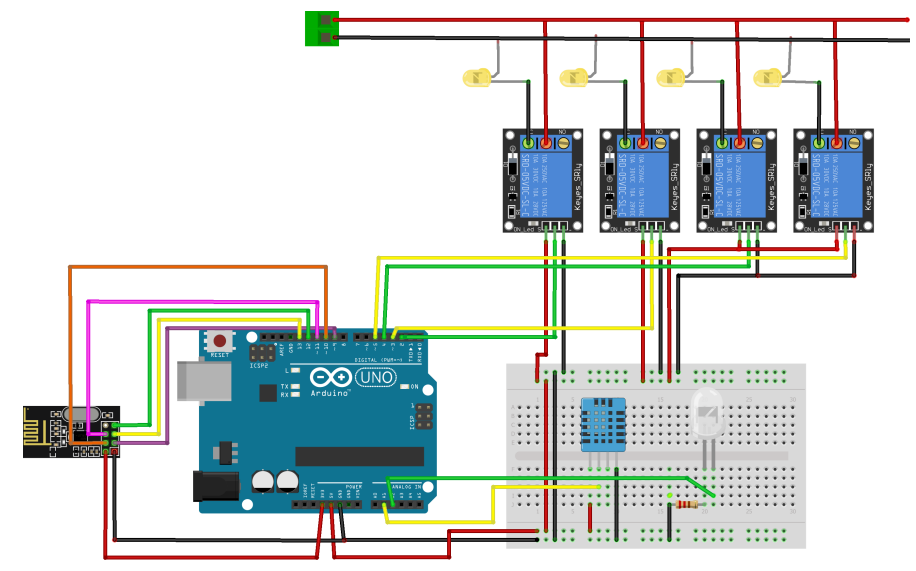

Figura 2. Nó 1, protótipo de sensoriamento da temperatura, umidade e iluminação, atuando no ambiente controlando as lâmpadas e arcondicionado.

O monitoramento do consumo da carga elétrica do ambiente é realizado pelo dispositivo apresentado na figura 3, desenvolvido por [Quindai et al. 2018], este nó será utilizado para medir o consumo da carga do ambiente que o sistema está inserido, composto por um Arduino UNO, um RTC (Real Time Clock) [Integrated 2015], um módulo NRF24L01 e três sensores de corrente não invasivos do tipo 100A SCR013.

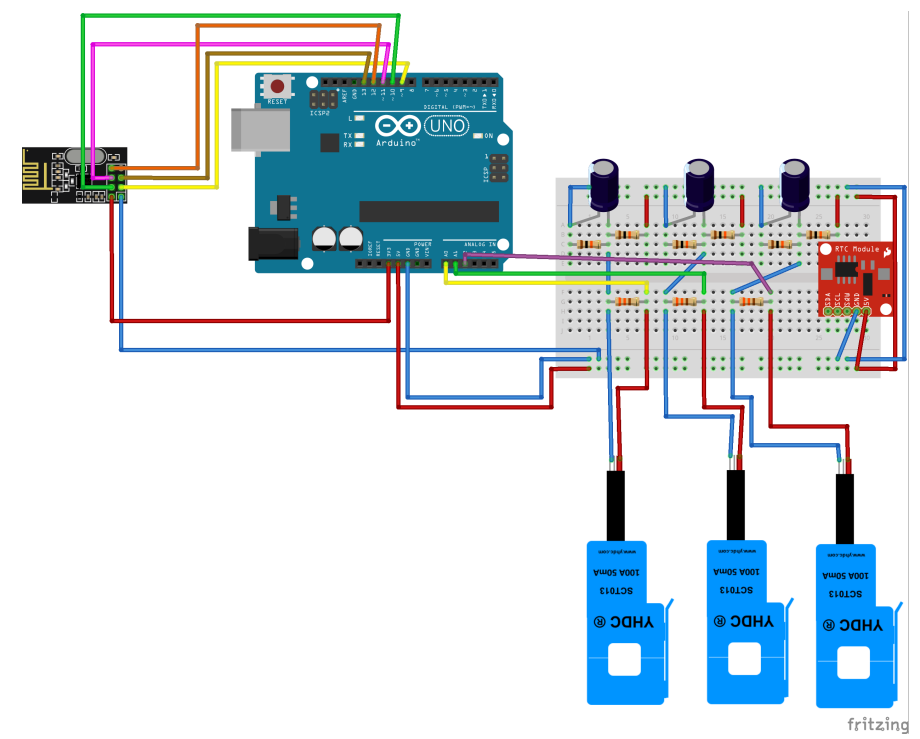

Figura 3. Nó 2, circuito de monitoramento trifásico da carga elétrica . 


\section{Resultados e Discussão}

O pré-processamento dos dados representa um ponto importante para que se obtenha um resultado de sucesso, e assim informações importantes para caracterização do ambiente possam ser extraídas. Apesar dos problemas relacionados a energia elétrica do ambiente sofre de constante falta de energia, o que gera grandes lacunas entre uma observação e outra, dificultando o armazenamento e rotulação de mais dados, conseguimos rotular 4 dias de dados entre os dias 20 e 24 de Outubro de 2018. A análise dos dados sofre com essa falta de informação, devido à dificuldade de se encontrar padrões que descrevam os dados, apesar disto, uma análise foi realizada em cima dos dados obtidos.

Conhecimentos obtidos anteriormente ajudando na mineração dos dados pelos autores através de sua vivência no ambiente são de suma importância para o preparamento dos dados. Estes estão relacionados a natureza do ambiente. Por ser um laboratório de pesquisa, é esperado um comportamento mais constante quando comparado a uma casa, apresentando uma maior mudança a cada seis meses quando o calendário de aulas e atividades são redefinidos dentro da universidade a qual este pertence.

Os dados de quando a sala está ou não ocupada foram capturados por uma câmera de vídeo, e foram rotulados visualmente, pois devido a utilização de uma técnica preditiva não supervisionada, necessitamos de uma base de dados para validação da técnica. Os dados dos sensores são enviados para o nó SINK a cada 30 segundos. Não houve nenhuma informação para os usuários que ambiente estava sendo monitorado, assim não interferindo na dinâmica habitual do uso da sala.

A tabela 1 apresenta uma amostra dos dados armazenados, com as seguintes descrições: ID, identificador do nó sensor; sensiron_temp, dados de temperatura em graus Celsius; sensiron hum, umidade relativa; light, incidência de iluminação no ambiente; date, data e hora do envio dos dados ao nó SINK onde subtrai-se três horas do horário coletado para representar a hora local; occupancy, identifica o status de ocupação da sala, ocupada representado por 1 e não ocupada por 0 .

Tabela 1. Amostra da representação dos dados monitorados pelos sensores.

\begin{tabular}{cccccc}
\hline ID & sensirion_temp & sensirion_hum & light & date & occupancy \\
\hline 6 & 22.8 & 54.6948 & 40.56 & $2018-10-2017: 07: 42$ & 0 \\
6 & 22.84 & 54.3575 & 40.46 & $2018-10-2017: 08: 12$ & 0 \\
6 & 33.67 & 54.0210 & 38.97 & $2018-10-2017: 08: 42$ & 0 \\
6 & 22.82 & 54.2743 & 38.87 & $2018-10-2017: 09: 12$ & 0 \\
\hline
\end{tabular}

Na figura 4, observamos que as informações começam no dia 21 de Outubro às 7:00 e terminam no dia 22 às 9:00, com isso podemos analisar um dia de comportamento dos usuários e a ocupação da sala neste período. Verificamos que existe uma diminuição da temperatura quando o ambiente está ocupado, isto ocorre pois o ar-condicionado é ligado pelos usuário, também podemos ver uma elevação da luminosidade devido a ligação das lâmpadas e ação da luminosidade externa da luz solar. A umidade também possui uma elevação quando o ar-condicionado e ligados e pessoas estão presentes no ambiente, por fim a série temporal que representa a ocupação na sala 1 caso esteja ocupada e 0 caso não. 


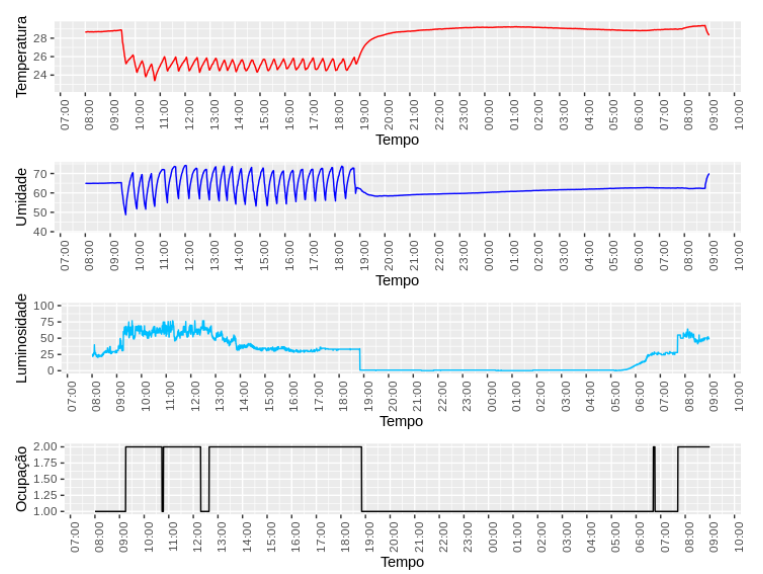

Figura 4. Série temporal dos dados sensoreados no período de 21 de outubro a 22 de 2018.

A figura 5 mostra um gráfico de pares, mostrando o relacionamento para todas as variáveis monitoradas. Como pode ser visto nos dados de temperatura e umidade, não há limites de separação clara para os pontos azuis e verdes, no entanto, as combinações de pares de temperatura e luz, mostram uma tendência de separação clara, o que indica que essa combinação de par são boas candidatas para treinar os modelos de classificação.

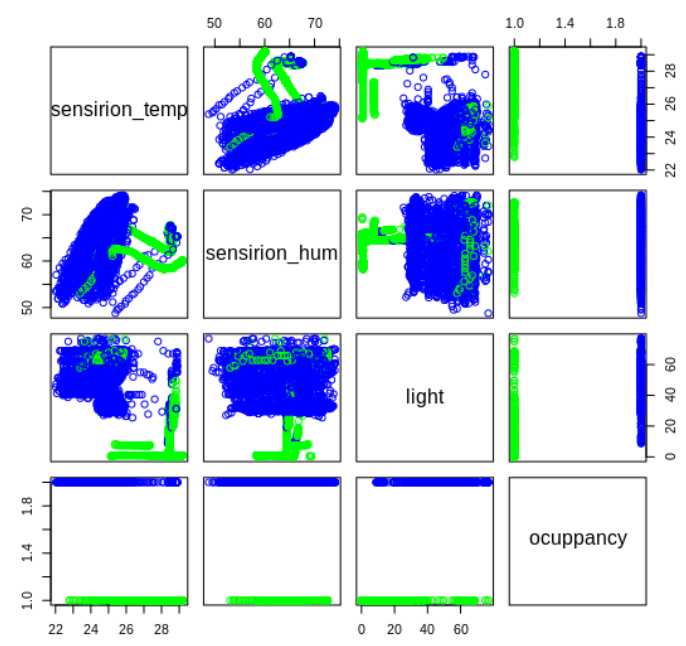

Figura 5. Pares de Correlação dos dados sensoreados pelo sistema.

Para o problema da ocupação, utilizamos as técnicas de classificação RF, CART e K-NN em nossos dados e aplicamos as mesmas métricas de avaliação das técnicas utilizadas por [Candanedo and Feldheim 2016] com intuito de comparação.

Calculamos a importância das variáveis quando utilizamos o modelo RF, essa importância é calculada usando o índice de Gini [Hastie et al. 2017], observamos que a variável sensiron_hum tem pouca ou quase nenhuma influência no modelo, indicando que a retirada desta variável irá melhorar o resultado da técnica.

Como pode ser observado na tabela 2 , os melhores resultados foram obtidos para 
as técnicas RF em ambas as bases de dados, onde utilizamos apenas dois preditores temperatura e luminosidade, $T$ e $L$ respectivamente.

Tabela 2. Performance dos modelos RF e CART, com resultados de [Candanedo and Feldheim 2016] $x$ e os dados da nossa aplicação $y$.

\begin{tabular}{lcccc}
\hline Modelo & Base & Parâmetros & Acurácia treinamento & Acurácia teste \\
\hline RF & $x$ & $T$ e $L$ & 99.85 & 95.93 \\
CART & $x$ & $T$ e $L$ & 99.08 & 95.43 \\
RF & $y$ & $T$ e $L$ & 99.85 & 98.50 \\
CART & $y$ & $T$ e $L$ & 96.65 & 95.62 \\
\hline
\end{tabular}

Aplicando K-NN em nossos dados com $k=2$ obtemos uma acurácia de $99.83 \%$. Podemos verificar na tabela 3 a matriz de confusão onde foram encontrados 4 valores falsos positivos significando que modelo previu que a sala estava ocupada mas não estava, e apenas dois falsos negativo, indicando que a sala estava vazia quando não estava. Utilizamos validação cruzada com o métodos $k$-fold que consiste em dividir o conjunto total de dados em $k$ subconjuntos mutuamente exclusivos do mesmo tamanho a cada iteração, uma das $k$ partições é usada para testar o modelo, as outras k-1 são usadas para treinar o modelo. Também verificamos excelentes taxas de F1-score, Precision e Recall.

Tabela 3. Matriz de Confusão e metricas de avaliação.

\begin{tabular}{|c|c|c|c|c|}
\hline \multicolumn{3}{|c|}{ Condição } & & \\
\hline previsão & 0 & 1 & F-1 Score & 99.86 \\
\hline 0 & 2186 & 4 & Precision & 99.90 \\
\hline 1 & 2 & 1368 & Recall & 99.81 \\
\hline
\end{tabular}

Para treinar o modelo gastou-se um tempo de 5.77 segundos em um computador com as seguintes configurações: intel core 17-7500U, memoria RAM DE 16GB; e um tempo de 12.01 segundos na RaspBerry (nó SINK). A análise e aplicação das técnicas de classificação foram realizadas utilizando a biblioteca $C A R E T^{4}$ e a linguagem de programação $R^{5}$.

\section{Conclusão}

Pode-se observar que a dinâmica da sala se modifica ao longo do dia, mostrando a necessidade de uma análise diária dos dados. Os dados de temperatura e luminosidade mostram uma tendência de separação, indicando uma baixa correlação entre eles. Também verificamos que a utilização da técnica K-NN com $K=2$, obteve um bom desempenho para o problema de classificação se a sala está ou não ocupada, em comparação com CART e RF. Por não precisar de muito tempo para treinar o modelo na Raspberry Pi 3, verificamos a facilidade de implementação da técnica em sistemas embarcados. A utilização da detecção de pessoas pode gerar uma redução significativa do consumo de energia para os ambientes monitorados onde o sistema proposto está instalado.

\footnotetext{
${ }^{4}$ https://github.com/topepo/caret/

${ }^{5}$ https://www.r-project.org/
} 
Outras aplicações podem ser testadas no contexto de SmartOffice, como conforto térmico, ajudando ainda mais na redução do consumo elétrico, aumentando a eficiência energética e o conforto dos usuários, tornando o sistema mais sensível ao contexto.

Por fim, para trabalhos futuros, iremos coletar mais dados do ambiente para outras realizar aplicações de outras técnicas de aprendizagem de máquina, assim tornar o ambiente mais sensível ao contexto, junto a uma análise do consumo de energia elétrica na sala, observando os resultados com o sistema implementado e sem o sistema.

\section{Referências}

Banavar, G., Morris, R., Naphade, M., Harrison, C., and Paraszczak, J. (2011). Smarter cities and their innovation challenges. Computer, 44:32-39.

Candanedo, L. M. and Feldheim, V. (2016). Accurate occupancy detection of an office room from light, temperature, humidity and $\mathrm{co} 2$ measurements using statistical learning models. Energy and Buildings, 112:28-39.

EPE - Empresa de Pesquisa Energética (2017). Anuário Estatístico de Energia Elétrica 2017, volume 1. http://www.eia.gov/totalenergy/data/annual.

Ghai, S. K., Thanayankizil, L. V., Seetharam, D. P., and Chakraborty, D. (2012). Occupancy detection in commercial buildings using opportunistic context sources. In 2012 IEEE International Conference on Pervasive Computing and Communications Workshops.

Hastie, T., Tibshirani, R., and Friedman, J. (2017). The Elements of Statistical Learning. Springer, second edition.

Integrated, M. (2015). Ds1307 64 x 8, serial, i2 c real-time clock.

Kleiminger, W., Beckel, C., Staake, T., and Santini, S. (2013). Occupancy detection from electricity consumption data. Proceedings of the 5th ACM Workshop on Embedded Systems For Energy-Efficient Buildings, pages 1-8.

Lantz, B. (2015). Machine Learning with R. Packt Publishing Ltd, second edition.

Ltd., A. E. (2010). Dht11 humidity e temperature sensor.

Nikolaou, T. G., Kolokotsa, D. S., Stavrakakis, G. S., and Skias, I. D. (2012). On the application of clustering techniques for office buildings' energy and thermal comfort classification. IEEE Transactions on Smart Grid, 3(4):2196 - 2210.

Nordic semiconductor (2008). nrfl24101+ prelimirary product specification.

Quindai, R., Barbosa, B. M., Almeida, C. M. P., Ramos, H. S., Rodrigues, J. J. P. C., and Aquino, A. L. L. (2018). A non-intrusive low-cost kit for electric power measuring and energy disaggregation. Journal of Communications Software and Systems, 14(1):9-17.

Technologies, S. (2010). Light dependent resistor ldr.

United Nations (2014). World Urbanization Prospects 2014: Highlights. Department of Economic and Social Affairs. United Nations Environment Programme. 